\title{
Portable and Low Power Efficient Pre-Fall Detection Methodology
}

\author{
Neeraj Rathi, Monika Kakani, Maher Rizkalla and Mohamed El-Sharkawy \\ Department of Electrical and Computer Engineering \\ Purdue School of Engineering, IUPUI \\ Indianapolis, United States \\ Email: mrizkalla@iupui.edu
}

\begin{abstract}
Fall in recent years have become a potential threat to elder generation. It occurs because of side effects of medication, lack of physical activities, limited vision, and poor mobility. Looking at the problems faced by people and cost of treatment after falling, it is of high importance to develop a system that will help in detecting the fall before it occurs. Over the years, this has influenced researchers to pursue the development to automatic fall detection system. However, much of existing work achieved a hardware system to detect pre and post fall patterns, the existing systems deficient in achieving low power consumption, user-friendly hardware implementation and high precision on a single portable system. This research points towards the development of dependable and low power embedded system device with easy to wear capabilities and optimal sensor structure. The designed system is triggered on interrupts from motion sensor to monitor users balanced, and unbalanced states. The fall decision parameters; pitch, roll, Signal Vector Magnitude (SVM), and Signal Magnitude Area (SMA) are layered to classify subject's different body posture. When the fall flag is set, the device sends important information like GPS location and fall type to caretaker. Early fall detection gives milliseconds of time to initiates the preventive measures. Near $100 \%$ sensitivity, $96 \%$ accuracy, and $95 \%$ specificity for fall detection were measured. The system can detect Front, Back, Side and Stair fall with consumption of $100 \mathrm{uA}(650 \mathrm{uA}$ with BLE consumption) in deep sleep mode, $6.5 \mathrm{~mA}$ in active mode with no fall, and $14.5 \mathrm{~mA}$, of which $8.5 \mathrm{~mA}$ is consumed via the BLE when fall is declared in active mode.
\end{abstract} device;

Keywords-pre-impact fall detection; Low power; Portable

\section{INTRODUCTION}

Falls in elder generation has become the frequent cause of injuries, physical disability, and death. According to World Health Origination (WHO) [1], fall is second leading cause of unintentional injury death after road accidents. Each year approximately 646000 individuals die from fall globally, in addition, 37.3 million falls are severe enough to require medical attention [1]. Regardless of extensive fall prevention programs [1], the number of falls and medical care is estimated to increase every year. Un-expected fall become dangerous for a person living alone. Staying with fall injuries for a long time may lead to hypothermia, rhabdomyolysis or dehydration. The study in [2] concludes that fall is an important factor of admittance into nursing home and therefore the system that prevent fall needs to be implemented to delay or reduce the chances to admittance into nursing home. Therefore, the need of a device that finds preventive measure to unexpected fall is necessary.

The importance of detecting and preventing a fall is unavoidable. Many researchers have also considered this issue and enormous work has been done to design a fall detection system with different approaches. In every related research, the authors have tried to overcome the existing research or proposed new technique to identify falls. Researchers have worked to develop systems with different categories, but the fall detection principles are either based on detecting fall before it happens know as Pre-Fall or detecting a fall after it has taken place known as after impact fall. The fall detection techniques found in literature are designed with both wearable devices and non-wearable devices.

The designed approaches have several advantages over one another, but all majorly lack in meeting low power requirements and standalone system, in addition, they are complex. The Pre-Fall detection system proposed by $\mathrm{Wu}$ [3] and Liu [4] was based on threshold-based fall detection using inertial sensors. The system works by continuous monitoring of user activity and logging the data. Such utilization of CPU results in high power consumption and reduces the comfortability of portable device. Also, fall decision based on single sensor often results in high false alarms. Shan [5] approached for Pre-Fall detection using machine learning. The system was contentiously measuring data and transmitting it to $\mathrm{PC}$ to provide fall information. The transmission of data to PC was done wireless hence compromising battery life by huge factor. In addition, the portability of system was a concern because of the complex machine learning algorithms. The method proposed by Leone [6] uses probes at different body location making it uncomfortable to use on daily basis. In addition, the device is not standalone; it requires external $\mathrm{PC}$ to process data. In [7-13] fall detection devices detects fall once it has taken place. Such system can only inform about fall but will not be able to take any preventive measure. [10-11] used smart phones to detect fall detection. The approach successfully reduces the hardware requirements but decreases the efficiency. Apart from the portable system, the nonwearable system described in [8] [12-13] has issue with cost, range, and efficiency. The vision-based system needs integration into surrounding which compromises the security, privacy and range. On the other hand, acoustic based approach provides less cost but has a noise issue. On contrary to nonwearable devices, the wearable devices developed using Micro-Electro-Mechanical Systems have advantage of being

This is the author's manuscript of the article published in final edited form as: 
available easily, less computation, smooth integration, and cheap. While using the wearable sensors, it is of importance to place them at right location on body. The body location of a sensor affects the detection capabilities [14].

\section{THE APPROACH}

Pre-Fall detection system in this research is designed to overcome the existing work in this field. The development of Pre-Fall detection system is done by considering real life scenarios, user flexibility and one's comfort towards using the wearable devices. The designed system completely integrates on a belt like structure, which can be easily wore around the waist area. As modern day embedded system must consume less power, provide high dependability, efficient communication, and low cost, the proposed system helps achieving the following:

- Automation: The developed device to detect fall event without any user interaction.

- Reliability: Distinguish fall event with other human daily activities.

- Power Consumption: Developed device switches between power modes to save on power.

- Low Power Wireless: Integrated with BLE capabilities to transmit fall information to care taker.

- Reaction time: The system detects Pre-Fall event approx. $250 \mathrm{~ms}$.

This work proposes a wearable sensor based on pre-fall detection system, which uses linear and angular velocity information from motion sensor to classify human fall. The sensors are integrated along with the controller on to a user's belt. The fall alert is shared to an android application developed for receiving fall information from measuring system. Research is also done to make sensor arranged in such a fashion that it gives high accurate data of human orientation with easy to wear on capabilities.

\section{A. System Architecture}

Figure 1 gives the overview of low power pre-fall detection system designed in our work. The system divides into sensing unit, controller unit and communication unit.

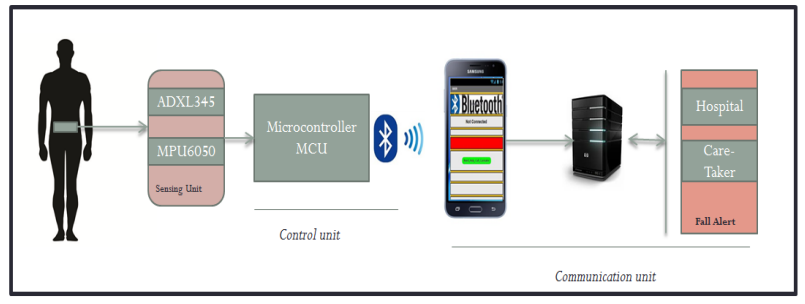

Fig. 1. System Overview

Sensing unit: The sensing unit consists of the activity monitoring sensors namely accelerometer and gyroscope. This unit is responsible for reliable data acquisition from ADXL345 and MPU6050 sensors mounted on the human body as a wearable technology.
Controlling unit: The controlling unit is the heart of the system. It consists of EFM32GG microcontroller and HM-10 BLE sensor. Its functionality includes initializing motion sensors, calibrating motion sensors, reading raw data, processing and computing raw data, analyzing computed data, and deciding about the fall.

Communication unit: The communication unit helps with transmitting the fall critical information to the caretaker, hospital, or health-care provider. On fall, the communication unit receives the information and transmits it to the caretaker in the form of a message with the time of fall and GPS location.

\section{B. Software Design Methodology}

Figure 2 shows the methodology of Pre-Fall detection. The approach is to sense high peaks in patient movements, which are caused by unstable actions, start of fall, and sudden sit. The high peak above the threshold generates an interrupt, waking up the MCU and MPU6050 to track human body posture for next two seconds. The window of two seconds is used to classify patient's activity after the peak. It also helps in minimizing unrecognized true-fall events. To ease our understanding on fall dynamics, the following events are defined.

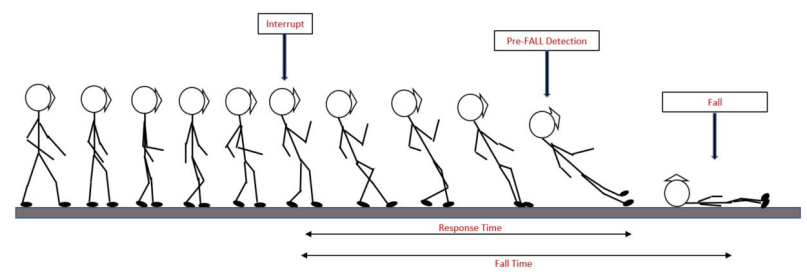

Fig. 2. Pre-fall Detection Approach

Interrupt Threshold: Motion thresholds are the acceleration peaks generated during a sudden sit, shock moments, freefalls, and rest. Detecting it gives a starting point of sudden activity or fall.

Fall Time: Fall time is the time when the interrupt is generated to the time when the fall happened.

Response Time: Response time is the time taken by the PreFall detection system to identify and declare fall since interrupt occurred.

Fall Detection: Detecting a fall at a posture from where the person cannot return to the normal or balance state. The fall detection decision in this work is truly based on thresholds.

$$
\text { PrefallTime }=\text { FallTime }- \text { ResponseTime }
$$

The equation 1 gives time at which the Pre-Fall detection system have detected a fall. This time helps us in designing a safety trigging system to protect patient.

\section{LOW POWER DESIGN AND EstimATION}

The low power approach in this work focuses on minimizing energy consumption of microcontroller based systems. The low voltage CMOS process have significantly contributed in lowering the power consumed by the micro- 
controller, but there is still need of work to design a low power system than just fabrication process . Our aim is to select ultralow power micro-controller and optimize the fall detection algorithm using the MCU features to extend battery life. The MCU consumes most power since it handles most arithmetic and logic operations, among others. Therefore, it is important to understand the cause of power consumption and steps to minimize it. Power consumption can be defined by Static and Dynamic power. The active state of CPU while processing the algorithm results into dynamic power consumption (ref equation 2).

$$
\text { Dynamic }_{\text {Power }}=V^{2} * f^{*} C
$$

$\mathrm{V}$ is voltage, $\mathrm{f}$ is frequency and $\mathrm{C}$ is load capacitance. Therefore, reducing the supply voltage and optimizing frequency helps in reducing dynamic power consumption.

Pre-Fall algorithm is designed to spend most of time in low power deep-sleep mode waiting for external interrupt-events to wake up the CPU. The power loses in deep sleep mode happens due to static power. While the code not actively running, the current consumption is due to bias currents for analog circuits, and leakage current. The static power can be reduced by using a MCU with designed advanced power management unit designed specifically to reduce leakage current. Other techniques used to reduce static power are disabling analog blocks, disabling ram blocks, and reducing supply voltage. The equation 3 shows total energy calculation.

Total $_{\text {Energy }}=$ ActiveMode $_{\text {Energy }}+$ DeepSleepMode Energy + Wakeup $_{\text {Energy }}$

Overall, the steps taken to reduce current consumption in active, deep sleep and wake up modes are: using energy modes, using low energy peripherals, disabling unused modules, optimizing clock, using lower operating voltage, and optimizing libraries.

\section{RESULTS AND DisCUSSION}

The test of the fall detection system has been conducted based on the hardware and software structure described above. The prototype was designed and tested in the lab environment. The data was analyzed from the test results gained from 3 females and 4 males subject's performing simulated falls and activity of daily living. The subject's age, body mass and height lie between $25-40$ years, $45-80 \mathrm{~kg}$, and $167-182 \mathrm{~cm}$ respectively. Figure 3 shows the designed system for Pre-Fall detection.

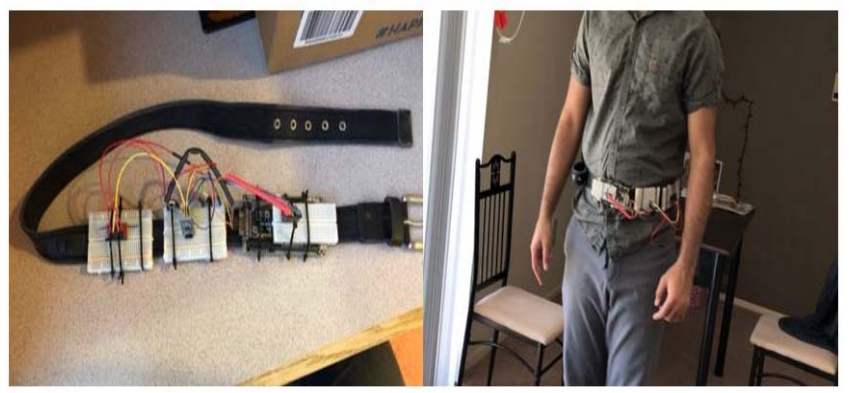

Fig. 3. The Practical Model

\section{A. The Mobile Application}

The Bluetooth mobile application was developed to receive fall signals from the detection system and send emergency message to caretaker. The Application utilizes the smart phone built in BLE, GSM and location sensor to receive and send message with GPS coordinates. It supports panic button to call and share location with care taker. Figure 4, shows the mobile application.

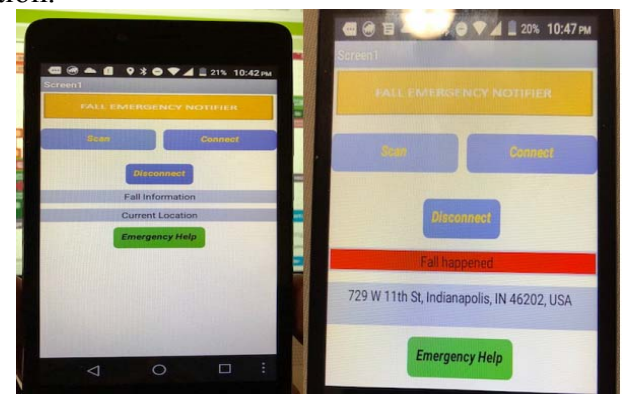

Fig. 4. The BLE Mobile Application

\section{B. Low Power Result}

The power consumption of the system: Microcontroller, MPU6050, ADXL345 and HM-10 is been monitored through Silicon labs Energy profiler. The current consumption was brought down to $\sim 600 \mu \mathrm{A}$ (HM-10: 510uA, ADXL345: 75uA, MCU:5uA, MPU6050: 10uA ) when processor in sleep mode and $\sim 6 \mathrm{~mA}$ during run mode. The current consumption gets higher when fall happens ( 14mA), because BLE sensor wakes up from sleep to send data. Figure 5 and 6 shows the optimized power consumption.

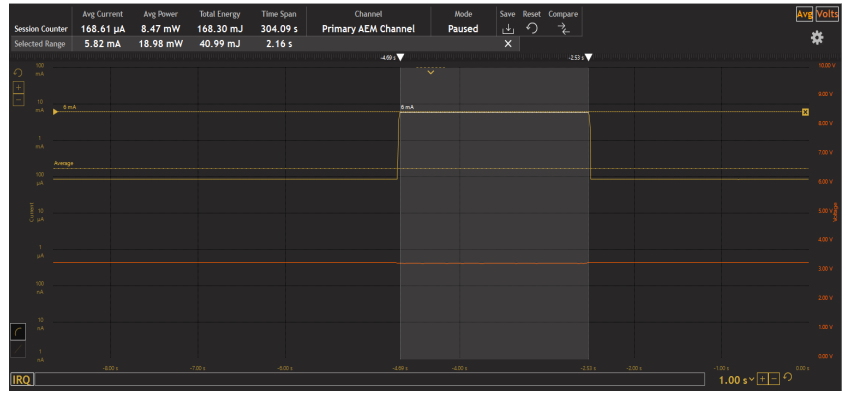

Fig. 5. The Power Consumption in Sleep and Active Mode

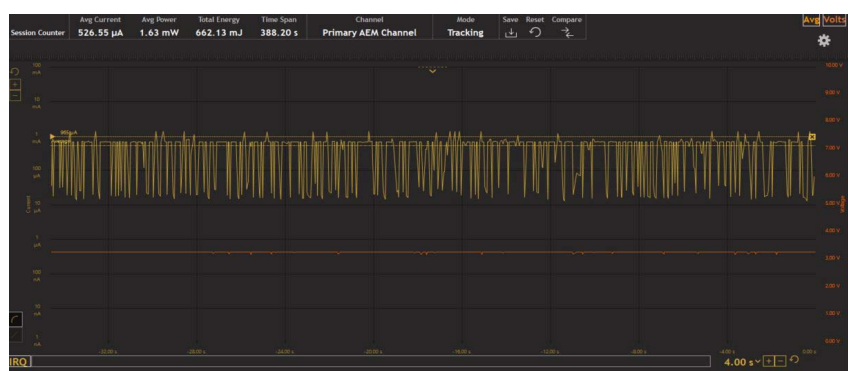

Fig. 6. The BLE Power Consumption in Sleep Mode

\section{Fall Parameters}

The patterns are acquired during walking, standing, sitting, and falling using the pitch and roll angles from low-pass filter 
and complimentary filter are shown in figure $7 \& 8$. Figure 9 shows the SVM and SMA result during back fall.

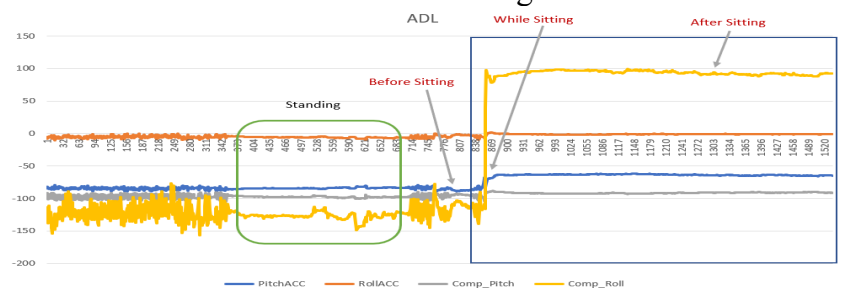

Fig. 7. Results Aquired While Performing Activity of Daily Living

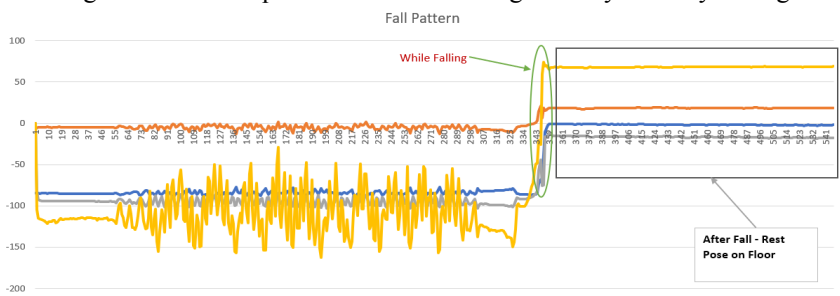

Fig. 8. Results Aquired While Performing Fall

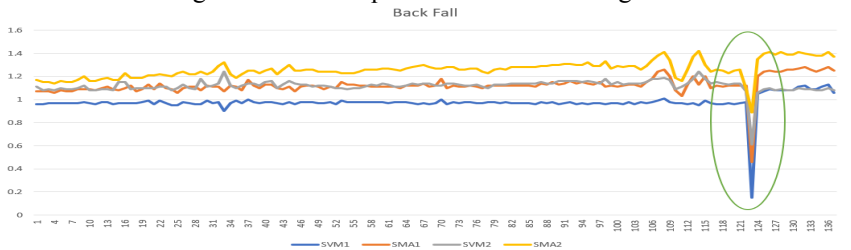

Fig. 9. SVM and SMA Data Aquired Duiring Back Fall

\section{System Test Result}

The results are obtained by performing test for four kinds of fall activity and ADL. Each fall and ADL posture has been repeated at least 15 times on each subject to determine thresholds. The accuracy, sensitivity, and specificity are determined using equations 4,5,6 from the test data in Table 1. The designed system estimates accuracy of $96.63 \%$, sensitivity of $100 \%$, and specificity of $95.45 \%$.

$$
\begin{gathered}
\text { Sensitivity }=\frac{T P F}{T P F+F N F} \\
\text { Specificity }=\frac{T N F}{F P F+T N F} \\
\text { Accuracy }=\frac{T P F+T N F}{T P F+F N F+F P F+T N F}
\end{gathered}
$$

Here, TPF is True Positive Fall, FNF is False Negative Fall, FPF is False Positive Fall, and TNF is True Negative Fall.

Table .1. Data Set

\begin{tabular}{|l|l|c|c|}
\hline \multirow{2}{*}{ Activity } & Incidents & True Positive Fall & False Negative Fall \\
\hline \multirow{3}{*}{ Human Fall } & Front Fall & 20 & 0 \\
\cline { 2 - 4 } & Back Fall & 15 & 0 \\
\cline { 2 - 4 } & Left-side Fall & 21 & 0 \\
\cline { 2 - 4 } & Right-side Fall & 21 & 0 \\
\hline \multirow{4}{*}{ ADL } & & False Positive Fall & True Negative Fall \\
\cline { 2 - 4 } & Sitting Down & 4 & 40 \\
\cline { 2 - 4 } & Standing Up & 1 & 70 \\
\cline { 2 - 4 } & Walking & 0 & 30 \\
\cline { 2 - 4 } & Resting & 5 & \\
\hline
\end{tabular}

\section{CONCLUSION}

In this work, a full characterization of the Pre-Fall detection system was conducted. This covered the hardware and software design, and the low power features. The practical results showed significant impact on the speed and power consumption. Near $250 \mathrm{~ms}$ was measured from the time of fall detection to the time when the fall occurs. The thresholdingbased classification model was used to differentiate between fall and ADL. The low power implementation was approached via the use of low power processor, the utilization of sleeping mode, and the software algorithm that featured low power.

\section{REFERENCES}

[1] "Falls," 2018 (accessed 15 February, 2018). [Online]. Available: http://www.who.int/mediacentre/factsheets/fs344/en/.

[2] M. E. Tinetti and C. S. Williams, "Falls, injuries due to falls, and the risk of admission to a nursing home," New England journal of medicine, vol. 337 , no. 18, pp. 1279-1284, 1997.

[3] G.Wu and S. Xue, "Portable preimpact fall detector with inertial sensors," IEEE Transactions on neural systems and Rehabilitation Engineering, vol. 16, no. 2, pp. 178-183, 2008.

[4] J. Liu and T. E. Lockhart, "Development and evaluation of a prior-toimpact fall event detection algorithm," IEEE Transactions on Biomedical Engineering, vol. 61, no. 7, pp. 2135-2140, 2014.

[5] S. Shan and T. Yuan, "A wearable pre-impact fall detector using feature selection and support vector machine," in IEEE 10th International Conference on Signal Processing, October 2010, pp. 1686-1689.

[6] A. Leone, G. Rescio, and P. Siciliano, "Fall risk evaluation by surface electromyography technology," 2017 International Conference on Engineering, Technology and Innovation (ICE/ITMC), pp. 1092-1095, 062017

[7] A. Jeza, E. Pramunanto, H. Boedinoegroho, and M. H. Purnomo, "Fall detection based on accelerometer and gyroscope using back propagation," in Electrical Engineering, Computer Science and Informatics (EECSI), 2017 4th International Conference. IEEE, 2017, pp. 1-6.

[8] S. Bhandari, N. Babar, P. Gupta, N. Shah, and S. Pujari, "A novel approach for fall detection in home environment," in 2017 IEEE 6th Global Conference on Consumer Electronics (GCCE), October 2017, pp. 1-5.

[9] M. Popescu and A. Mahnot, "Acoustic fall detection using one-class classifiers," in Engineering in Medicine and Biology Society, 2009. EMBC 2009. Annual International Conference of the IEEE. IEEE, 2009, pp. 3505-3508.

[10] Y. He, Y. Li, and S. D. Bao, "Fall detection by built-in tri-accelerometer of smartphone," in Proceedings of 2012 IEEE-EMBS International Conference on Biomedical and Health Informatics, January 2012, pp. 184-187.

[11] F. Sposaro and G. Tyson, lifall: an android application for fall monitoring and response," in Engineering in Medicine and Biology Society, 2009. EMBC 2009. Annual International Conference of the IEEE. IEEE, 2009, pp. 6119-6122.

[12] S. Gasparrini, E. Cippitelli, S. Spinsante, and E. Gambi, "A depth-based fall detection system using a kinect ${ }^{\circledR}$ sensor," Sensors, vol. 14, no. 2, pp. 2756-2775, 2014

[13] E. E. Stone and M. Skubic, "Fall detection in homes of older adults using the microsoft kinect," IEEE journal of biomedical and health informatics, vol. 19, no. 1, pp. 290-301, 2015.

[14] A. T. Ozdemir and B. Barshan, "Detecting falls with wearable sensors using machine learning techniques," Sensors, vol. 14, no. 6, pp. 10691$10708,2014$. 